\title{
A Cross Layer Protocol for Improving the Performance of Ad Hoc Networks
}

\author{
S.Vinoth Kumar $^{1}$, G.Selvakumar ${ }^{2}$
}

\author{
S.Vinoth kumar ${ }^{l}$, \\ M.E-Computer Science and Engineering, \\ Sri Shakthi Institute of Engineering \\ Technology, Coimbatore, India. \\ Anna university, Chennai.
}

\author{
Mr.G. Selvakumar ${ }^{2}$ \\ Assistant Professsor, \\ Sri Shakthi Institute of Engineering and \\ Technology, Coimbatore, India.
}

\begin{abstract}
To implement the cross layer protocol for improving the performance of multi-hop wireless network by routing, scheduling the transmission and traffic management. Unicast and multicast routes are established in coordination with the scheduling of transmissions and bandwidth reservations in a way that bandwidth and delay guarantees can be enforced on a per-hop and end-to-end basis. It establishes and maintains loop-free routes from sources to destinations. Traffic management is done by priority based queuing system. In addition, the routing algorithm establishes enclaves, which restrict the spread of control information to those nodes that are likely to participate as forwarders of a given data flow, rather than the entire network. Performance achieved by reducing the end-to-end delay, efficient packet delivery and reducing the communication overhead. By Simulation, comparing the existing protocols, this protocol attains better performance for elastic traffic, less delay and effective data delivery with less communication overhead. Further more, performance can be enhanced by scheduling the transmission more efficiently using token bucket filter scheme.
\end{abstract}

Index Terms-Cross Layer, Bandwidth Reservations, Traffic management, Enclaves, Token Bucket Filter

\section{INTRODUCTION}

An ad hoc network is a collection of wireless mobile nodes dynamically forming a temporary network without the use of existing network infra-structure. Due to the limited transmission range of wireless network interfaces, multiple network hops may be needed for one node to exchange data with another across the network. In such a network, each mobile node operate not only as a host but also as a router, forwarding packets for other mobile nodes in the network, that may not be within the direct reach wireless transmission range of each other. Each node participates in an ad hoc routing protocol that allows it to discover multi hop paths through the network to any other node. It is true that performance suffers as the number of devices grows and large ad-hoc networks become difficult to route and manage. However many protocols are introduced to improve the performance of ad hoc networks in large networks.

Section 2 provides a small sample of the existing protocols that includes scheduling mechanism, reservation protocols, routing protocols, cross layer designs to improve the performance of ad hoc networks. The existing protocols have focused on improving the performance of ad-hoc network.

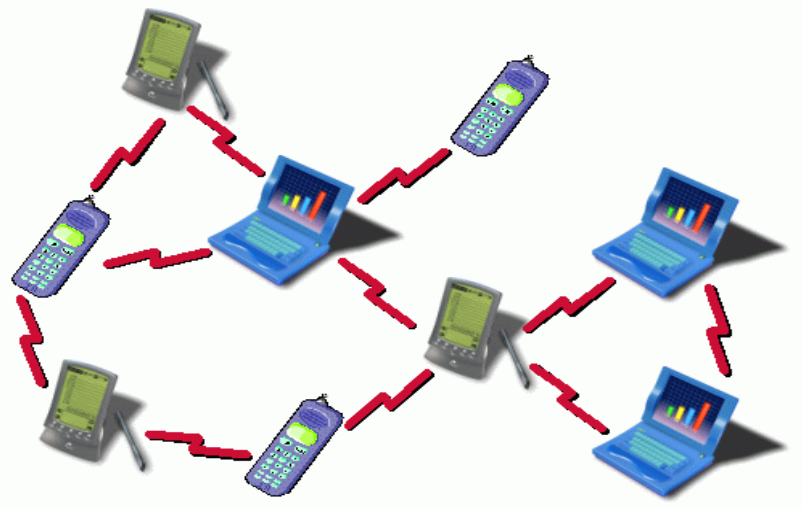


The solutions that have been proposed in the past are either based on centralized algorithms requiring too much information at each node, or do not combine routing and scheduling with the establishment of bandwidth reservations and traffic handling. Furthermore, prior solutions do not address the integration of unicast and multicast routing with transmission scheduling. No proposals have been made on the integration of scheduling and routing for many-to-many communication using distributed algorithms based on local information.

The main contribution of this paper is to introduce a new cross-layer protocol to combine routing with the scheduling and traffic handling. It supports both unicast and multicast routing. Sections 3 describe about the objective of this protocol. Section 4 describes the transmission scheduling and traffic handling in this cross layer protocol. This scheduling is to schedule the transmission for a node to access the channel. The traffic handling describes the priority-based queuing system used to handle signalling traffic, elastic data flows and real time flows. Section 5 describes the neighbour protocol which is used to provide information about the reservation of neighbour nodes. Section 6 describes the end-to-reservation scheme and the maintenance of end-to-end channel access schedule. Section 7 describes the routing mechanism in cross layer protocol. This protocol provides flow ordered routing meshes which consist of multiple paths from source to destination. Section 8 describes the processing and transmission of mesh statements which tells about processing of packets in a node and the procedures to transmit the packet to the next hop of routing meshes that connecting the destination in multi hop networks. Section 9 describes the results of simulation experiments used to study the performance of our protocol and compare it with the performance of existing protocols.

Our cross layer protocol provide some performance improvements even for both unicast and multicast because it avoids most packet collisions and limits the communication overhead needed for routing.

\section{RELATED WORK}

Many protocols are implemented in order to enhance the performance of ad hoc wireless networks. Some Existing protocols like AODV is introduced for the effective elastic traffic in wireless network and support unicast routing. However in AODV it does not reserves the bandwidth, Such that BW consumption is more, which affect the performance. So 802.11 DCF is introduced for effective routing by reserving the bandwidth and it reduces the delay. 802.11 DCF extends by reserving the bandwidth but it does not integrate routing with scheduling. So here the communication overhead is high. To overcome this, MACA/PR attempted to integrate routing and traffic management to improve the efficiency. But MACA/PR works only for unicast routing and reserved free slots which are not used will reduce the bandwidth efficiency.

DARE is a channel access protocol for MANETs that provides end-to-end reservations. It is based on request-to reserve messages that travel from sources to destinations through routes established by a traditional on-demand routing protocol. Destinations reply with clear-to-reserve messages that travel along reverse paths establishing the actual reservations. Data packets also contain reservation information and are used to refresh the reservations tables.The main limitations of DARE are that reservations are established at each hop of a path independently of the other hops in the path, and outing decisions do not consider information regarding reservations or any other data collected for channel access. DSDV that keeps track of the bandwidth of the shortest paths to each destination and the maximum bandwidth available over all possible paths. The first data packet of a real-time flow makes reservations along the path for subsequent packets in the connection. One-hop scheduling information is piggybacked in data packets and ACKs which reserve time-synchronized windows at specified time intervals. Reservations are made taking into account only two-hop neighbourhood information and without coordination with the routing protocol.

Some prior cross layering approaches attempting to make routing and channel access more efficient in ad hoc networks. Chen and Heinzelman provide a comprehensive survey on routing protocols that provide some sort of support for QoS in MANETs, and Melodia et al. present a survey of cross-layer protocols for wireless sensor networks. Setton et al. propose a cross-layer framework that incorporates adaptations across all layers of the protocol stack. The proposed framework, however, is mostly based on centralized algorithms and a link-state approach is needed, which is not well suited for the highly dynamic MANETs or very large ad hoc networks. In the context of multicast communication, most of the work has focused on static networks.In these works, the authors formulate the joint multicast routing and power control problem [22] or the network planning problem as a cross-layer optimization problem. However, no proposals have been made on the integration of scheduling and routing for many-to-many communication.

\section{CROSS LAYER PROTOCOL}

A cross-layer approach seeks to enhance the performance of Ad hoc wireless network. The objective of our cross layer protocol is to arrange scheduling, routing, and traffic management functions of a multi-hop wireless network so that sources and destinations of flows becomes very efficient for both unicast and multicast transmissions and the routes established are loop free. 


\section{TRANSMISSION SCHEDULING}

Scheduling done to know which node must transmit in a time slot. The node with its identifier first checks if it is the owner of the slot, the owner can access the channel. If the node does not own the slot, it checks if the owner is present in its two-hop neighbourhood and listen to the channel. If there is a collision, the time slot is not considered as owned by any of the nodes and a hash-based election is held among the nodes participating in the collision. If the owner of the time slot is not present in the two-hop neighbourhood, the node checks whether it has a reservation on the slot in which case it can access the channel. Otherwise it checks whether neighbour node has reserved the slot, if they reserved they can access the channel. If no two-hop neighbour nodes have reserved, hash based election scheme is done to select the node that can access the network.

Traffic Handling: This uses reservations and a priority-based queuing system to implement and maintain the per-flow channel access schedules. Nodes reserve time slots for data flows according to their end-to-end schedules. When a node is allowed to transmit over a time slot, it has many packets in it. Packets are selected from the local transmission queues, which are FIFO and are served using a priority-based algorithm. Reserved packets have the highest priority. The next priority is given to network-layer signaling packets and data packets waiting in data queues have the lowest priority. Data queues can be either elastic or real-time, and real-time queues are assigned higher priority than the priority given to elastic queues.

\section{NEIGHBOUR PROTOCOL}

The neighbour protocol is also used to detect when two nodes in a two-hop neighbourhood have reserved the same slot. To resolve a conflicting reservation, the node with the larger identifier keeps its reservation over the particular slot, whereas the node with the lower identifier has to give up its current reservation and start a new reservation transaction over a different slot. The main source of these conflicting reservations is node mobility, which changes the neighbourhood of nodes. The neighbourhood information contained in hello messages allows nodes to detect these collisions before the conflicting nodes become one-hop neighbours.

A free slot for node is a slot not currently owned or reserved by any node in its two hop neighbourhood, including $\mathrm{x}$ itself, and that is not in the process of being reserved. The information needed to verify these conditions is stored in three data structures, namely, the Neighbour Lists, the Ongoing Reservation Lists, and the Reserved Slot List. The Neighbour Lists stores information regarding the identity of nodes and reserved slots within the two-hop neighbourhood and is maintained by the Neighbour Protocol.

\section{END-TO-END RESERVATION}

When a node starts transmitting real-time data packets for a source-destination pair, it reserve future slots to be used on that particular real-time flow. the selection of a particular slot is controlled by the routing so that the channel access schedules of the relays of the flow are flow ordered. The relays are flow ordered if every single one of them can access the channel in a time-ordered sequence of slots. The node that is relaying data packets toward destination compute the interval of flow-ordered slot identifiers corresponding to its current distance to destination. By reserving slots from these intervals, any two consecutive nodes that lay in a path of routing meshes will have the right to access the channel. In addition to the reservation of slots, a node that becomes part of a real-time flow has to create a new real time queue associated with the flow and with the set of slots reserved on that flow. This protocol guarantees that the bandwidth reserved on behalf of a real-time flow is actually used by that data flow, and that different flows that traverse the same node do not interfere with each other breaking the per-flow ordering of the end-to-end reservations.

During a real-time flow, nodes must ensure that the end-to-end sequence of reservations is maintained, even in the mobility of nodes. When a node actively transmitting the packet to the next hop or destination, if the node found that its distance to the destination changes, they cancel all the reservation and request a new set of reservations. In this protocol, nodes wont cancel the reservation immediately for the changes in distance, instead the nodes will verify whether the changes is stable or not.

\section{ROUTING}

The routing in our cross layer protocol supports both unicast and multicast transmission. The routes established is shown to be loop free. Routing in this protocol is based on destination nodes, routing meshes, and enclaves. Routing meshes is used to connect the source and the destination. Each node in those path of routing meshes is flow ordered in the real time flow, by the order of end-to-end reservation of time slots. Enclaves is used to avoid the spreading of control information to the nodes which are participating to forward the packets to the destination. 
In multi-hop wireless networks, the source sends its first data packet piggybacked in a Mesh Request (MR) to the forwarder or intermediate node. Thus packet receivers of an MR start the process of establishing and maintaining its routing mesh and enclave. The MR packet must contain information about sender's address, destination address, distance from the sender and limiting the control information.

The routing meshes is maintained to co-ordinate the end-to-schedule in the flow ordered real time flow. Routing meshes are maintained by mesh statements(MS) transmitted by the forwarder to the destination or next hop. The Mesh Statement (MS) will have the information about forwarder's address, destination or preferred next hop's address, distance from the forwarder, largest sequence number known by forwarder and the flag indicating that the forwarding node is flow ordered. The routing meshes provide a fast and efficient way of repairing routes, because they contain extra paths that can be used in case of link breaks. This reduces the impact of mobility of nodes on quality of service in real time flow.

Each node in multi-hop network maintains neighbourhood list that stores set of MS's received from the neighbours. The list also keep tracking of information about largest sequence number, current distance to the destination and next hop's address. Thus the routes established shown to be loop free.

\section{PROCESSING AND TRANSMISSION OF MESH STATEMENTS}

The node accept the MS sent by the forwarder only when the current sequence number is equal or larger stored in forwarder node. The current distance to the destination can be reset by new sequence number. The current distance to the destination can be measured by the number of hops connecting to destination. The address of the next hop can be computed by the current values of distance to destination, sequence number and flow ordered flag.

A node transmits MSs to inform other nodes about updates in its routing state. The updates made by changes in the intermediate nodes (that join or leave the group) and so the new sequence number is generated. Whenever the new larger sequence number generated the next hop pointer to the destination is established.

Packet Forwarding: The node forwards a data packet received from previous node if that node selected by the previous node as one of its next hops to the destination. Data packets travel along routing meshes until they reach the destination. Realtime data packets are routed composed of multiple flow-ordered paths. This way, nodes adapt their end-to-end schedules.

\section{SIMULATION RESULTS}

\section{a. Latency}

As a first performance metric, we look at the latency of packets in a real-time flow. The term latency is an expression for the period of time taken to send a packet from a source to the intended destination. Higher the latency, the slower the data transmission. Graph shows in terms of the latency, comparing our cross layer protocol with existing protocol. The implemented new cross layer protocol has less latency manages to deliver all packets of reserved flows to their destination within less time. On the other hand, existing protocol deliver a substantially smaller fraction of packets within this time; Increasing the number of real-time flows, makes the differences between these protocols even more pronounced. The delay is predictable when knowing the number of hops that a packet has to travel.

\section{b. packet received}

The next graph shows the comparison of our protocol with the existing protocol in terms of number of packet received per unit time. More number of packets are received in our protocol, thus our protocol suits best for large networks.

\section{c. packet delivery ratio}

Packet delivery ratio is defined as the ratio of data packets received by the destinations to those generated by the sources .This performance metric gives us an idea of how well the protocol is performing of packet delivery at different speeds using different traffic models. From the graph we see that our protocol has gained higher percentage of packet delivery ratio than the existing protocol which leads to improvement in performance of network. 

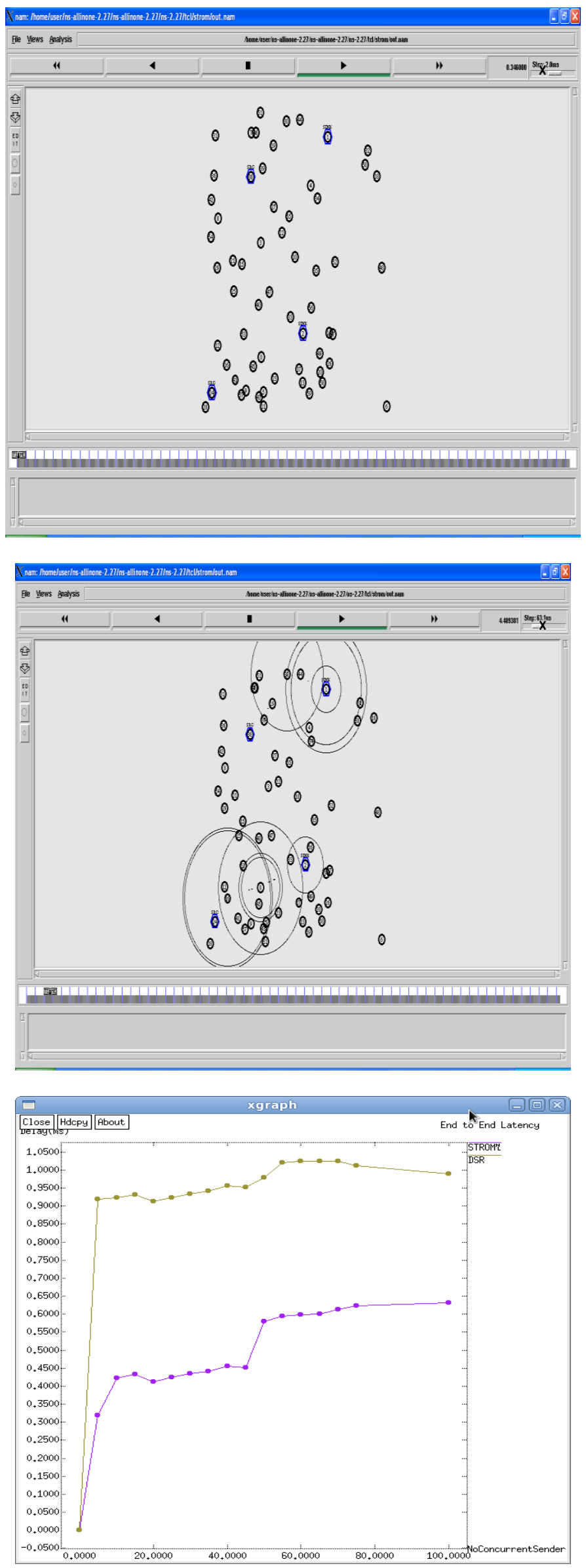

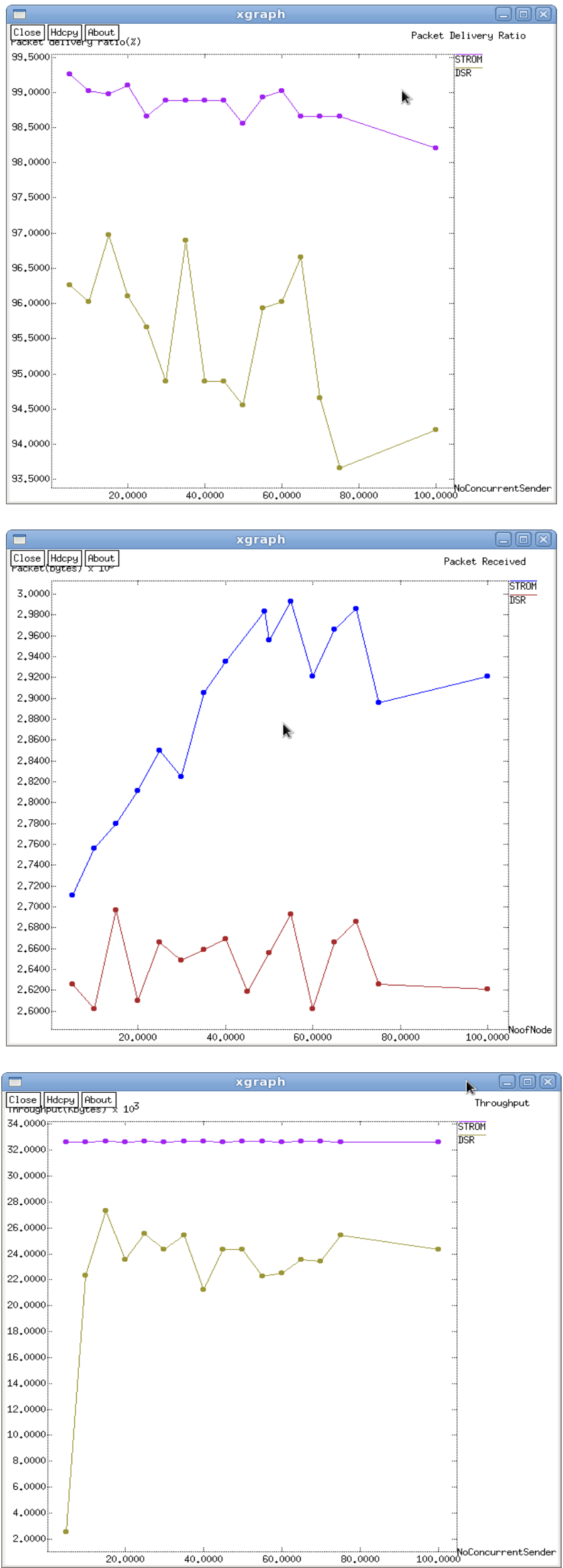


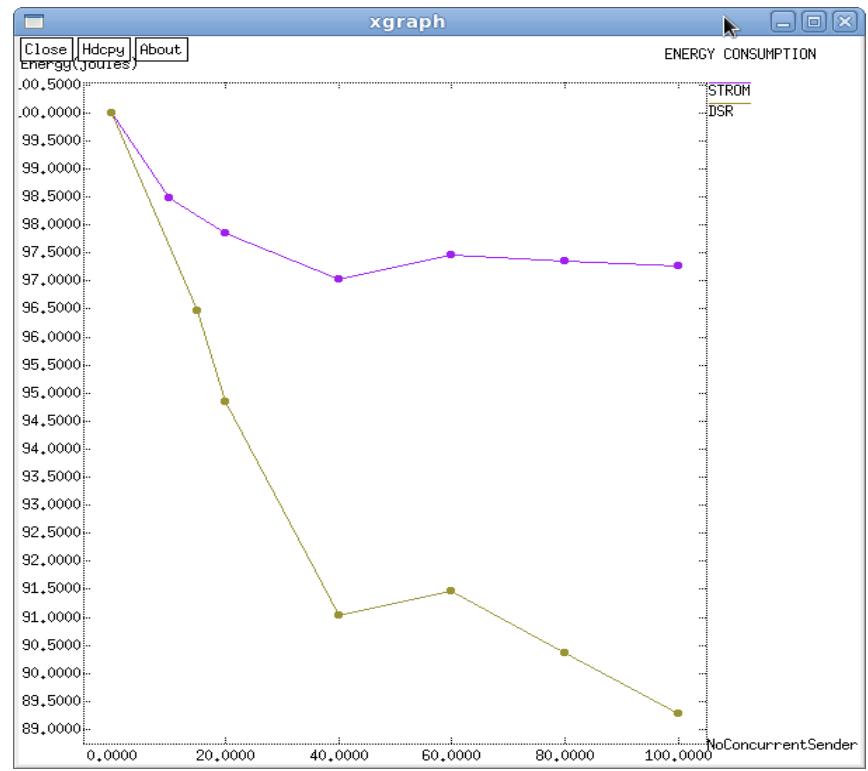

\section{CONCLUSION}

We introduced a cross-layer protocol for wireless ad hoc networks that combine routing with prioritybased queuing for traffic management, end-to-end bandwidth reservations controlled by the routing, and distributed transmission scheduling. All these mechanisms work together to provide unicast and multicast data flows in multi-hop wireless networks even when nodes move. We proved that the route established by this protocol are loop-free at any time and that the end-to-end reservations established along routing meshes provide bounded delays to real-time data packets. Also scheduling done by token bucket filter scheme to use the bandwidth efficiently. Our cross layer protocol is very scalable and robust for both unicast and multicast traffic.

Our simulation-based study shows that our protocol provides high packet delivery ratio and less delay as well as less overhead. While the performance of existing approaches strongly degrades with increasing network load, our protocol performs well even for high traffic loads.

\section{REFERENCES}

1). E. Carlson, C. Prehofer, C. Bettstetter, H. Karl, and A. Wolisz, "ADistributed End-to-End Reservation Protocol for IEEE 802.11-Based Wireless Mesh Networks,” IEEE J. Selected Areas in Comm.,vol. 24, no. 11, pp. 2018-2027, Nov. 2006.

2). L. Baoand J.J. Garcia-Luna-Aceves, "A New Approach to ChannelAccess Scheduling for Ad Hoc Networks,” Proc. ACM MobiCom,pp. 210-221, 2001.

3). R. Menchaca-Mendez and J.J. Garcia-Luna-Aceves, "An Interest-Driven Approach to Integrated Unicast and MulticastRouting in MANETs," Proc. IEEE 16th IEEE Int'l Conf. NetworkProtocols (ICNP '08), pp. 248-257, Oct. 2008.

4). E. Setton, T. Yoo, X. Zhu, A. Goldsmith, and B. Girod, "CrosslayerDesign of Ad Hoc Networks for Real-Time Video Streaming,’IEEE Wireless Comm., vol. 12, no. 4, pp. 59-65, Aug. 2005.

5). C.E. Perkins and P. Bhagwat, "Highly Dynamic Destination Sequenced Distance-Vector Routing (DSDV) for Mobile Computers,"Proc. ACM SIGCOMM, pp. 234-244, 1994.

6). C.R. Lin and M. Gerla, "Asynchronous Multimedia MultihopWireless Networks," Proc. IEEE INFOCOM, vol. 1, pp. 118-125,Apr. 1997.

7). T. Melodia, M.C. Vuran, and D. Pompili, "The State of the Art inCross-Layer Design for Wireless Sensor Networks," Proc. EURONGIWorkshops Wireless and Mobility, pp. 78-92, 2006.

8). P. Djukic and P. Mohapatra, "Soft-TDMAC: Software TDMABasedMAC over Commodity 802.11 Hardware," Proc. IEEEINFOCOM, 2009.

9). L. Chen and W.B. Heinzelman, "A Survey of Routing Protocolsthat Support QoS in Mobile Ad Hoc Networks," IEEE Network,vol. 21, no. 6, pp. 30-38, Nov./Dec. 2007.

10). E. Setton, T. Yoo, X. Zhu, A. Goldsmith, and B. Girod, "CrosslayerDesign of Ad Hoc Networks for Real-Time Video Streaming,’IEEE Wireless Comm., vol. 12, no. 4, pp. 59-65, Aug. 2005. 